Pensamiento Crítico Vol. 19 № 1, pp. 85-104

\title{
La empleabilidad potencial de los estudiantes de la UNMSM
}

Employability potential of students of San Marcos

Juan León Mendoza*

Miguel Arias Inga

Richard Roca Garay

\section{RESUMEN}

El objetivo central de este artículo consiste en construir un índice de empleabilidad de los estudiantes de la UNMSM y, a su vez, identificar los factores que condicionan la probabilidad de que estos encuentren empleo.

El índice de empleabilidad potencial promedio de los estudiantes sanmarquinos es de $55.51 \%$, que representa una empleabilidad media.

El resultado de la regresión econométrica efectuada con el método del modelo probit indica que la probabilidad de que un alumno de la UNMSM consiga empleo o una práctica preprofesional es mayor en la medida en que sea mayor su promedio de notas ponderadas, sea responsable, maneje más programas informáticos, conozca los métodos de búsqueda de trabajo, posea experiencia laboral, tenga la percepción de que su perfil profesional es adecuado para lo que demanda el mercado y mantenga empatía con sus compañeros de la universidad.

Palabras clave: empleo, empleabilidad, empleabilidad estudiantil.

\footnotetext{
* Magíster en economía. Docente principal de la Facultad de Ciencias Económicas
} 


\title{
Pensamiento Crítico Vol. 19. $\mathrm{N}^{\circ}$ I
}

\begin{abstract}
This article aims to develop a UNMSM student population employability index by identifying the main conditioning factors affecting their probability of getting employed.

Thus, the results show that the mean potential employability index of the UNMSM students stands at $55.51 \%$.

The economical regression was made through the probit model method, and the traits assessed were their marks arithmetic mean, responsibility, ease of use of informatics software, job finding skills, work experience, the own perception of their resume to satisfy adequately the general requirements of the market and, overall empathy with their colleagues. We found that there exists a clear correlation with being over the average at this traits and a higher chance of getting employed.
\end{abstract}

Keywords: Job, employability, student employability

\section{Introducción}

La Universidad Nacional Mayor de San Marcos, como una casa superior de estudios está relacionada con la formación de profesionales, por lo que su flujo de jóvenes egresados también enfrenta retos y dificultades en su proceso de inserción en el mercado laboral. Pero, dicha inserción estará condicionada, en parte, por la calidad de los conocimientos que haya asimilado o acumulado durante su permanencia en la universidad, es decir, por la capacidad adquirida para ser empleable.

Por ello, se hace relevante las siguientes preguntas, que trataremos de responder en el presente artículo:

¿Cuál es el grado de empleabilidad potencial de los estudiantes de la Universidad Nacional Mayor de San Marcos en general? ¿Qué variables influyen en la probabilidad de encontrar empleo?

\section{Marco Teórico}

Existen muchas definiciones sobre el concepto de empleabilidad, por lo que no se tiene una única definición internacionalmente aceptada; incluso se tienen diferentes indicadores para su medición (Forrier y Sels, 2003). 


\section{Juan León Mendoza, Miguel Arias Inga, Richard Roca Garay}

En este escenario, la Organización Internacional del Trabajo (OIT) define la empleabilidad como: “... uno de los resultados fundamentales de una educación y formación de alta calidad y de la ejecución de varias otras políticas. Abarca las calificaciones, conocimientos y las competencias que aumentan la capacidad de los trabajadores para conseguir y conservar un empleo..."1.

En esta definición sobresale la importancia que tiene para la empleabilidad de las personas los conocimientos adquiridos mediante una educación de calidad. La educación es un elemento muy importante en la acumulación de capital humano, es decir, del stock de conocimientos y habilidades humanas.

La formación universitaria está directamente involucrada en la formación de aptitudes. Sin embargo, una educación superior de calidad también puede contribuir a crear en el estudiante actitudes que lo hagan más competitivo y empleable. En tal sentido, también se puede entender empleabilidad como la capacidad de las instituciones educativas de desarrollar en el estudiante el potencial para conseguir y conservar empleo.

La definición del concepto de empleabilidad está en función de la operacionalización necesaria para generar indicadores de empleabilidad correspondientes a determinados tipos de investigación. En tal sentido, se hace relevante la siguiente definición dada por Harvey $(1999)^{2}$ acerca de la empleabilidad de los jóvenes que culminaron su educación superior: "... la propensión del graduado de exhibir atributos que los empleadores anticipan que sean necesarios para el efectivo funcionamiento futuro de su organización".

Sin embargo, un aspecto importante para la empleabilidad es que el joven desee trabajar y tome la decisión de buscarla. Porque podría tener los atributos necesarios, pero, si no busca empleo es muy poco probable que lo consiga por ende su empleabilidad tiende a cero (Rodríguez, 2009). Por eso, algunos investigadores sostienen que las actitudes, junto con los conocimientos y competencias personales, condicionan la empleabilidad (Rodríguez et. al., 2010).

1 Citada por Unión Europea (2010)

2 Citada por Rodríguez (2009) 


\section{Pensamiento Crítico Vol. 19. No I}

Corpoeducación (2003) sostiene que las competencias son la base para lograr el mejoramiento de las condiciones de empleabilidad de las personas. Dichas competencias pueden ser básicas, ciudadanas y laborales. A su vez, las competencias laborales pueden ser generales o específicas.

Según A/B (2010), los elementos que determinan la empleabilidad de la persona se pueden clasificar en siete grupos de factores: personales, laborales de la empresa, del entorno socioeconómico, educativos académicos, profesionales, sociolaborales e institucionales. Entre ellas destaca la triada persona-empresa-sociedad.

En el plano de las investigaciones empíricas, Puente y Rau (2010) efectuaron una regresión probit para determinar qué factores condicionaban la probabilidad de conseguir empleo en Estados Unidos de Norteamérica. Se consideró como factores explicativos el sexo, la edad, área de residencia del individuo. En tanto que como variable dependiente se consideró una variable binaria: se le dio un valor de cero si el individuo estaba desempleado y un valor de uno si estaba empleado. Se encontró que todas las variables consideradas en la regresión tenían una importancia explicativa en la probabilidad de conseguir empleo.

Rentería y Andrade (2007), en un estudio efectuado entre los estudiantes de tres Facultades de una universidad pública de Cali-Colombia, destacan la importancia que le dan los estudiantes al establecimiento de redes y la tenencia de experiencia laboral para incrementar sus empleabilidad. Además, queda en un rango de mucha menor importancia el saber un segundo idioma y la presentación personal.

Según Rodríguez (2009), la empleabilidad de los profesionales -que depende significativamente de la calidad de la formación recibida en los centros de educación superior debe ser medida también en términos potenciales, en tanto que los futuros egresados son estudiantes. El análisis de la empleabilidad potencial permite no solo conocer las competencias que están adquiriendo los estudiantes, sino que también permite identificar los problemas que se va teniendo en su proceso de formación. Adicionalmente, el estudio de la empleabilidad potencial coadyuva a determinar el perfil de los estudiantes, lo cual puede ser un elemento muy importante para reajustar la currícula de estudios. 


\section{Juan León Mendoza, Miguel Arias Inga, Richard Roca Garay}

Rodríguez op. cit. construyó un índice de empleabilidad potencial de los estudiantes del Instituto de Educación Superior Chaminade Marianistas del Callao, considerando indicadores correspondientes a trece aspectos: capacitación, motivación, redes sociales, educación, salud, acceso a TIC, economía, sociales, habilidades, competencias, información, currículum vitae y la experiencia laboral. En ella encuentra que estos aspectos son relevantes para la construcción de un índice de empleabilidad potencial.

\section{Aspectos metodológicos}

Elaboraremos el índice de empleabilidad potencial de los estudiantes de la UNMSM, tomando como referencia básica las dimensiones de empleabilidad propuestas por Rodríguez (2009), Allen et. al. (2003), Collado (2006) y Fundación Gaztelán (2007). Para ello, hemos considerado ocho dimensiones. Cada una de las dimensiones contiene indicadores relevantes específicos, con su correspondiente puntaje máximo, tal como se pueden observar en los siguientes ocho cuadros:

\section{CUADRO No 1 . COMPETENCIAS PERSONALES}

\begin{tabular}{|l|l|c|}
\hline \multicolumn{1}{|c|}{ COMPETENCIAS PERSONALES } & \multicolumn{1}{|c|}{ INDICADOR } & \multicolumn{1}{c|}{$\begin{array}{c}\text { PUNTAJE } \\
\text { MÁXIMO }\end{array}$} \\
\hline Competencia capacidad para aprender & $\begin{array}{l}\text { Las veces que estudia un artículo de su } \\
\text { especialidad para entenderlo }\end{array}$ & 3 \\
\hline Competencia flexibilidad o movilidad & Disponibilidad de trabajar en cualquier lugar & 2 \\
\hline Competencia emprendimiento & $\begin{array}{l}\text { Disponibilidad de cambio de trabajo y } \\
\text { emprendimiento }\end{array}$ & 2 \\
\hline Competencia persuasión & Capacidad de persuadir a los demás & 1 \\
\hline Competencia trabajo en equipo & Si le es cómodo trabajar en equipo o solo & 3 \\
\hline Competencia liderazgo & Si ocupó cargos de dirigencia estudiantil & 3 \\
\hline Competencia empatía & $\begin{array}{l}\text { Percepción de la calidad personal de sus } \\
\text { compañeros de estudio }\end{array}$ & 1 \\
\hline Competencia comunicación & $\begin{array}{l}\text { Proporción de sus compañeros con los que } \\
\text { mantiene una comunicación fluida }\end{array}$ & $\begin{array}{l}\text { Si pertenece a algún círculo de estudios o de } \\
\text { investigación }\end{array}$ \\
\hline Personalidad desarrollo de relaciones & $\begin{array}{l}\text { Veces en que llegó tarde a sus clases en la } \\
\text { universidad en el último semestre }\end{array}$ & 2 \\
\hline Actitud de puntualidad & & \\
\hline
\end{tabular}




\section{Pensamiento Crítico Vol. 19. $\mathrm{N}^{\circ}$ I}

\begin{tabular}{|l|l|c|}
\hline Actitud iniciativa & Percepción de capacidad de iniciativa & 2 \\
\hline Actitud perseverancia & Percepción de capacidad de perseverancia & 2 \\
\hline Valores integridad & $\begin{array}{l}\text { Actitud ante una pregunta de examen cuya } \\
\text { respuesta desconoce }\end{array}$ & 2 \\
\hline Personalidad autocontrol & $\begin{array}{l}\text { La manera de reaccionar ante una ofensa } \\
\text { personal }\end{array}$ & 2 \\
\hline Personalidad tolerancia a la presión & Si le gusta o incomoda estudiar bajo presión & 2 \\
\hline Personalidad confianza en sí mismo & $\begin{array}{l}\text { Su grado de nerviosismo ante dificultades en } \\
\text { las evaluaciones }\end{array}$ & 1 \\
\hline Competencia de manejo automotor & Si posee licencia de conducir & 2 \\
\hline
\end{tabular}

\section{CUADRO No 2. EDUCACIÓN}

\begin{tabular}{|l|l|c|}
\hline \multicolumn{1}{|c|}{ EDUCACIÓN } & \multicolumn{1}{|c|}{ INDICADOR } & $\begin{array}{c}\text { PUNTAJE } \\
\text { MÁXIMO }\end{array}$ \\
\hline Récord académico & $\begin{array}{l}\text { Promedio de notas obtenido en el último } \\
\text { semestre académico }\end{array}$ & 4 \\
\hline $\begin{array}{l}\text { Estudio de otro programa o carrera } \\
\text { profesional }\end{array}$ & Si estudió otra carrera profesional o técnica & 1 \\
\hline Dominio de programas informáticos & $\begin{array}{l}\text { El número de programas informáticos que } \\
\text { domina (diferentes al Word, Excel y Power } \\
\text { Point) }\end{array}$ & 4 \\
\hline
\end{tabular}

\section{CUADRO No 3 . CAPACITACIÓN}

\begin{tabular}{|l|l|c|}
\hline \multicolumn{1}{|c|}{ CAPACITACIÓN } & \multicolumn{1}{|c|}{ INDICADOR } & $\begin{array}{c}\text { PUNTAJE } \\
\text { MÁXIMO }\end{array}$ \\
\hline Participación en cursos de capacitación & $\begin{array}{l}\text { Número de cursos de capacitación certificados } \\
\text { en el último año }\end{array}$ & 3 \\
\hline Participación en conferencias y seminarios & $\begin{array}{l}\text { Número de seminarios o conferencias en los } \\
\text { últimos tres meses }\end{array}$ & 3 \\
\hline
\end{tabular}

\section{CUADRO $N^{\circ}$ 4. HABILIDADES BÁSICAS}

\begin{tabular}{|l|l|c|}
\hline \multicolumn{1}{|c|}{ HABILIDADES BÁSICAS } & \multicolumn{1}{c|}{ INDICADOR } & $\begin{array}{c}\text { PUNTAJE } \\
\text { MÁXIMO }\end{array}$ \\
\hline Redacción & Percepción de capacidad de redacción & 3 \\
\hline Idiomas & El grado de dominio del idioma inglés & 2 \\
\hline
\end{tabular}




\section{Juan León Mendoza, Miguel Arias Inga, Richard Roca Garay}

\section{CUADRO No 5. MOTIVACIÓN}

\begin{tabular}{|l|l|c|}
\hline \multicolumn{1}{|c|}{ MOTIVACIÓN } & \multicolumn{1}{|c|}{ INDICADOR } & $\begin{array}{c}\text { PUNTAJE } \\
\text { MÁXIMO }\end{array}$ \\
\hline $\begin{array}{l}\text { Conocimiento de métodos de } \\
\text { búsqueda de trabajo }\end{array}$ & Si conoce los métodos de búsqueda de trabajo & 1 \\
\hline $\begin{array}{l}\text { Percepción de oportunidades } \\
\text { laborales }\end{array}$ & $\begin{array}{l}\text { Percepción del nivel de la demanda en el } \\
\text { mercado de trabajo por profesionales de su } \\
\text { especialidad }\end{array}$ & 3 \\
\hline Compatibilidad de perfil laboral & $\begin{array}{l}\text { Percepción de grado de compatibilidad de } \\
\text { su perfil profesional con lo que usualmente } \\
\text { demanda el mercado }\end{array}$ & 1 \\
\hline Consulta de fuentes de empleo & $\begin{array}{l}\text { Si consultó fuentes de empleo en los últimos } \\
\text { tres meses }\end{array}$ & 1 \\
\hline Envío de currículum vitae & $\begin{array}{l}\text { Si envió currículum en la búsqueda de trabajo } \\
\text { o de prácticas preprofesionales en los últimos } \\
\text { tres meses }\end{array}$ & \\
\hline
\end{tabular}

\section{CUADRO No 6. REDES SOCIALES}

\begin{tabular}{|l|l|c|}
\hline \multicolumn{1}{|c|}{ REDES SOCIALES } & \multicolumn{1}{|c|}{ INDICADOR } & $\begin{array}{c}\text { PUNTAJE } \\
\text { MÁXIMO }\end{array}$ \\
\hline Figuración en redes sociales & Si está en linkedin & 1 \\
\hline Cantidad de contactos & $\begin{array}{l}\text { Cantidad de contactos con que se cuenta para } \\
\text { la consecución de empleo }\end{array}$ & 3 \\
\hline $\begin{array}{l}\text { Pertenencia a algún club social o } \\
\text { cultural }\end{array}$ & Si es miembro de algún club social y cultural & 1 \\
\hline
\end{tabular}

\section{CUADRO $\mathbf{N}^{\circ}$ 7. ASPECTOS LABORALES}

\begin{tabular}{|l|l|c|}
\hline \multicolumn{1}{|c|}{ ASPECTOS LABORALES } & \multicolumn{1}{|c|}{ INDICADOR } & $\begin{array}{c}\text { PUNTAJE } \\
\text { MÁXIMO }\end{array}$ \\
\hline Situación laboral actual & $\begin{array}{l}\text { Si actualmente trabaja, practica o solo } \\
\text { estudia }\end{array}$ & 3 \\
\hline Experiencia laboral & $\begin{array}{l}\text { Si trabajó o efectuó prácticas pre } \\
\text { profesionales en años anteriores }\end{array}$ & 1 \\
\hline
\end{tabular}




\section{Pensamiento Crítico Vol. 19. No I}

\section{CUADRO N 8. FACTORES SOCIOECONÓMICOS}

\begin{tabular}{|c|c|c|}
\hline FACTORES SOCIOECONÓMICOS & INDICADOR & $\begin{array}{l}\text { PUNTAJE } \\
\text { MÁXIMO }\end{array}$ \\
\hline $\begin{array}{l}\text { Calidad de educación secundaria } \\
\text { recibida }\end{array}$ & $\begin{array}{l}\text { Si estudió la educación secundaria en un } \\
\text { colegio privado o público }\end{array}$ & 1 \\
\hline Nivel educativo del padre & Nivel educativo alcanzado por el padre & 3 \\
\hline Posesión de vivienda & Si cuenta o no con vivienda propia & 1 \\
\hline Disponibilidad de computadora & $\begin{array}{l}\text { Si cuenta o no con una computadora } \\
\text { personal }\end{array}$ & 1 \\
\hline Disponibilidad de internet & Si cuenta con servicio domiciliario de internet & 1 \\
\hline Disponibilidad de pasaporte & Si tiene o no pasaporte & 1 \\
\hline Nivel socioeconómico & Distrito de Lima y Callao en que reside & 3 \\
\hline
\end{tabular}

Los indicadores que muestran un puntaje máximo de uno son variables binarias. Toma el valor de cero la alternativa que corresponde al que no contribuye -o contribuye menos- a la empleabilidad y toma el puntaje de uno el que contribuye -o contribuye en mayor grado- a la empleabilidad.

En el mismo sentido, aquellos indicadores con valores máximos de tres o cuatro corresponden al número de alternativas que contienen. Todos tienen un valor mínimo de cero y su valor sube en la medida en que el estudiante muestre indicadores que coadyuven en un mayor grado a la empleabilidad.

En total, se tienen 41 indicadores de empleabilidad, siendo el puntaje máximo total igual a 82. El índice de empleabilidad total puede tomar valores que van de 0 a 100. Un índice de 100 equivale al puntaje máximo total de 82. De acuerdo al puntaje alcanzado, el estudiante individual es clasificado en una de las cinco categorías de empleabilidad: muy alta empleabilidad (100-77 puntos), alta empleabilidad (76-63 puntos), empleabilidad media (50-62 puntos), empleabilidad baja (30-49 puntos) y muy baja empleabilidad (menor de 29 puntos).

Una vez calculado el índice de empleabilidad para cada estudiante encuestado, el índice de empleabilidad de una facultad se estima mediante un promedio simple de los índices de empleabilidad hallados para los estudiantes de la facultad correspondiente. El índice de empleabilidad de los estudiantes de la UNMSM, en conjunto, se calcula 


\section{Juan León Mendoza, Miguel Arias Inga, Richard Roca Garay}

mediante la suma ponderada de los índices de empleabilidad de las 20 facultades que existen en la UNMSM.

Con la finalidad de especificar las variables que determinan la probabilidad de hallar empleo o prácticas preprofesionales, efectuamos una regresión econométrica utilizando el método del modelo probit.

El universo de la investigación comprende a todos los estudiantes matriculados de la UNMSM. Se efectuaron 461 encuestas válidas a aquellos matriculados en el último año de su formación profesional y que, por ende, estaban ad portas de buscar trabajo, estaban buscando trabajo o, ya venían trabajando.

\section{Características de la empleabilidad}

A continuación mostramos los resultados de la encuesta sobre las características, comprendidas en las ocho dimensiones de empleabilidad, que presentan los estudiantes de la UNMSM. Se debe advertir que dichas características se especifican sobre la base de la opinión dada por los encuestados y, es generalizable a toda la población estudiantil de la UNMSM, en la medida en que la encuesta se aplicó a una muestra estadísticamente representativa.

Relacionado a la dimensión de competencias personales, el $56 \%$ de los estudiantes encuestados indican que estudian 2 veces, en tanto que un $30 \%$ manifiestan que lo hacen 3 veces, solo un $8 \%$ señala que lo hace por una sola vez. Por otro lado, el $51 \%$ está dispuesto a buscar trabajo y laborar en el extranjero y un $34 \%$ en provincias fuera de Lima; solo un $15 \%$ está dispuesto a buscar trabajo en la capital peruana. En el hipotético caso de que ya tienen buen empleo y están remunerados satisfactoriamente, el $46 \%$ está dispuesto a emprender una empresa o negocio propio.

El 54\% de los encuestados cree poseer una mediana capacidad de persuasión y un $43 \%$ una alta capacidad en dicha competencia personal. El $84 \%$ prefiere desarrollar las tareas o trabajos en grupo; solo un $16 \%$ prefiere hacerlo solo. Si bien la preferencia por trabajar en equipo es alta, sin embargo, solo un $25 \%$ manifiesta pertenecer a círculo de estudios o de investigación universitaria. El $62 \%$ nunca ejerció cargo dirigencial alguno, lo hizo sólo el $32 \%$. Un $56 \%$ opina que sus compañeros de estudio en su mayoría son 


\section{Pensamiento Crítico Vol. 19. N I}

excelentes personas. Un $52 \%$ manifiesta que mantiene una fluida comunicación con la mayoría de sus compañeros de estudio. En el semestre académico anterior, un $45 \%$ de los estudiantes llegó tarde muchas veces a sus sesiones de clase en la universidad, un $52 \%$ lo hizo pocas veces. Sin embargo, solo el 3\% nunca llegó tarde. Esta cifra indica la predominancia de la irresponsabilidad, por ende, es un indicador negativo para la empleabilidad potencial del estudiante sanmarquino.

Un $62 \%$ manifiesta poseer una moderada capacidad de iniciativa personal; solo el $36 \%$ indica que tienen una virtud de alta iniciativa. El $82 \%$ indica que siempre practica la perseverancia para alcanzar sus objetivos personales. El $70 \%$ manifiesta que casi siempre no escribe nada en un examen escrito si no conoce la respuesta a la pregunta formulada. Solo el 24\% manifiesta que sí escribe algo (relativamente no son honestos). Ante una ofensa recibida, el $82 \%$ responde de una manera calmada y un $13 \%$ indica ignorarlo. El $61 \%$ prefiere estudiar o trabajar bajo presión, en tanto que un $30 \%$ es adverso a dicha presión. Ante situaciones de alta dificultad en los procesos de evaluación que enfrenta, el $77 \%$ manifiesta sentirse un poco nervioso, un $8 \%$ indica sentirse muy nervioso. Solo el $10 \%$ de los encuestados posee la competencia formal de conducir vehículos (cuenta con brevete), por lo que una mayoría predominante de $90 \%$ no tiene dicha competencia.

Respecto a la dimensión de la educación, el $42 \%$ de los encuestados manifiesta haber obtenido un promedio de calificación de 13-14 en el semestre académico anterior, el $35 \%$ obtuvo entre 15-16; solo el $16 \%$ tuvo una calificación mayor de 16 . Por otro lado, un $64 \%$ manifiesta no haber estudiado un programa o una carrera aparte de la especialidad que está estudiando en la UNMSM. Un 69\% manifiesta conocer y manejar más de dos paquetes informáticos diferentes al Office (Word, Excel, POWER POINT)..

En lo que se refiere a la dimensión de capacitación, en el último año, un 35\% de ellos no asistieron a ningún curso de capacitación, el 25\% asistió a un curso, un $21 \%$ a dos cursos y un $20 \%$ a más de dos cursos. Por otro lado, el $31 \%$ de los encuestados manifiestan no haber asistido a ningún tipo de este certamen en los últimos tres meses; asistió una vez el $26 \%$, dos veces el $20 \%$ y tres veces el $23 \%$. 


\section{Juan León Mendoza, Miguel Arias Inga, Richard Roca Garay}

En referencia a la dimensión de las habilidades básicas, el 63\% de los estudiantes manifiestan que tienen una buena capacidad de redacción y un $25 \%$ una capacidad regular; aquellos que sostienen tener una muy buena redacción representan apenas el $1 \%$. Solo el $29 \%$ de los estudiantes manifiestan dominar el idioma inglés, en tanto que el $55 \%$ lo domina un poco, habiendo un porcentaje de $16 \%$ que no lo sabe.

Relacionado a la dimensión de la motivación, el 75\% de los estudiantes encuestados manifiestan conocer los métodos de búsqueda de trabajo, en tanto que el $25 \%$ manifiesta no conocer ningún método. El 59\% percibe que en el mercado laboral existe una regular demanda por los profesionales de su especialidad, en tanto que el $32 \%$ cree que la demanda es muy poca. Es prácticamente nula la percepción de que existe mucha demanda; en este marco poco optimista, existe un $9 \%$ que cree que no existe demanda.

El $49 \%$ percibe que su currículum personal calza poco con lo que demanda el mercado de trabajo. Solo el $32 \%$ cree que calza bastante, existiendo un $5 \%$ que cree que existe un desencuentro total entre su currículum personal y las disponibilidades de trabajo en el mercado laboral. Por otro lado, el $52 \%$ de los estudiantes manifestaron haber consultado alguna fuente de empleo en los últimos tres meses, en tanto que el $48 \%$ no lo hizo. En la búsqueda de empleo o prácticas preprofesionales, en los últimos tres meses, solo el $43 \%$ de los estudiantes enviaron su currículum correspondiente, en tanto que el $57 \%$ no lo hizo.

Correspondiente a la dimensión de redes sociales, el 65\% de los alumnos encuestados indican que están registrados en la red virtual de contactos profesionales Linkedin, pero un sorprendente $35 \%$ manifiesta no estarlo. Por otro lado, un mayoritario $56 \%$ manifiesta contar con muy pocos familiares, amigos o conocidos que le puedan servir de contacto para conseguir trabajo y un $30 \%$ indica que cuenta con una regular cantidad de contactos; el porcentaje de aquellos que no cuentan con ningún contacto es relativamente baja (7\%). Finalmente, el $77 \%$ indica que no pertenece o no es miembro de algún grupo social o cultural, en tanto que un porcentaje minoritario de $23 \%$ manifiesta que sí lo es. 


\section{Pensamiento Crítico Vol. 19. N I}

En referencia a la dimensión de los aspectos laborales, en el escenario en que la encuesta se aplicó solo a los alumnos que están a puertas de egresar de la universidad, del total de estudiantes encuestados, el $76 \%$ ya trabaja o que están efectuando prácticas pre profesionales, en tanto que el $24 \%$ no trabaja ni practica. El $69 \%$ indica que ya cuenta con experiencia laboral o que ha efectuado prácticas pre profesionales, sólo un minoritario $31 \%$ no tiene dicha experiencia.

Finalmente, respecto a la octava dimensión de empleabilidad sobre los aspectos socioeconómicos, la mayoría de los estudiantes encuestados $(62 \%)$ estudiaron la educación secundaria en un colegio estatal. Para un 53\% su padre posee educación superior. La mayoría de ellos cuenta con vivienda propia (76\%), poseen computadora personal (93\%) y disponen del servicio de Internet en casa (90\%). Apenas el $16 \%$ cuenta con pasaporte.

\section{5. Índice de empleabilidad potencial}

El índice de empleabilidad potencial de los estudiantes de la Universidad Nacional Mayor de San Marcos en general es de 55.51, lo cual indica que el estudiante sanmarquino presenta, en promedio, una empleabilidad media. Dicho resultado también indica que los estudiantes sanmarquinos, en promedio, no presentan una alta empleabilidad.

A nivel de facultades tampoco se tienen casos de empleabilidad alta. Todas, sin excepción, están comprendidas en la categoría de empleabilidad media.

Sin embargo, existen diferencias en el grado de empleabilidad entre los estudiantes de las 20 facultades de la UNMSM. La Facultad de Ingeniería Geológica, Minera, Metalúrgica y Geográfica muestra el índice más alto de empleabilidad potencial, con un puntaje de 60.42. Posteriormente, entre las cuatro facultades con los mejores índices relativos de empleabilidad están Ingeniería Industrial, Ciencias Biológicas e Ingeniería de Sistemas, tal como se puede observar en el cuadro $\mathrm{N}^{\circ} .9$.

La facultad que muestra el más bajo índice relativo de empleabilidad potencial es la de Odontología. Además, entre el grupo de las cuatro Facultades con los menores índices estar Ciencias Físicas, Química e Ingeniería Química y, Letras y Ciencias Humanas. 
Juan León Mendoza, Miguel Arias Inga, Richard Roca Garay

\section{CUADRO No 9. ÍNDICE DE EMPLEABILIDAD POTENCIAL DE LOS ESTUDIANTES DE LA UNMSM}

\begin{tabular}{|l|c|}
\hline \multicolumn{1}{|c|}{ FACULTAD } & ÍNDICE \\
\hline Ing. Geológica, Minera, Metal. y Geográfica & 60.42 \\
\hline Ingeniería Industrial & 60.39 \\
\hline Ciencias Biológicas & 59.68 \\
\hline Ingeniería de Sistemas & 59.04 \\
\hline Medicina Veterinaria & 58.74 \\
\hline Ciencias Administrativas & 58.16 \\
\hline Farmacia y Bioquímica & 57.89 \\
\hline Ingeniería Electrónica y Eléctrica & 57.23 \\
\hline Psicología & 57.19 \\
\hline Derecho y Ciencia Política & 56.93 \\
\hline Ciencias Económicas & 55.54 \\
\hline Educación & 54.59 \\
\hline Ciencias Sociales & 53.56 \\
\hline Ciencias Contables & 53.29 \\
\hline Ciencias Matemáticas & 53.07 \\
\hline Medicina & 52.66 \\
\hline Letras y Ciencias Humanas & 52.40 \\
\hline Química e Ingeniería Química & 52.13 \\
\hline Ciencias Físicas & 51.39 \\
\hline Odontología & 50.98 \\
\hline PROMEDIO PONDERADO & 55.51 \\
\hline
\end{tabular}

Elaboración propia 


\section{Pensamiento Crítico Vol. 19. No I}

\section{Determinantes de la probabilidad de conseguir empleo}

Con la finalidad de identificar las variables que determinan la probabilidad de que el estudiante sanmarquino consiga o no empleo, a continuación efectuamos las regresiones correspondientes utilizando el método del modelo probit.

Después de un ejercicio de iteración automática, el modelo econométrico final a regresionar fue el siguiente:

$$
\begin{gathered}
\mathrm{y}_{\mathrm{i}}=\alpha_{0}+\alpha_{1} \text { COMP }_{\mathrm{i}}+\alpha_{2} \text { EXPLAB }_{\mathrm{i}}+\alpha_{3} \text { PERFPRO }_{\mathrm{i}}+\alpha_{4} \text { MBTRA }_{\mathrm{i}}+\alpha_{5} \text { INFORM }_{\mathrm{i}} \\
+\alpha_{6} \text { EMPAT }_{\mathrm{i}}+\alpha_{7} \text { NOT }_{\mathrm{i}}+\alpha_{8} \text { RESPON }_{\mathrm{i}}+\mathrm{u}_{\mathrm{i}}
\end{gathered}
$$

Donde:

$$
y_{i}=\left\{\begin{array}{l}
1=\text { Si el estudiante está trabajando o ef ectuando prácticas pre profesionales } \\
0=\text { Si el estudiante no trabaja o no efectúa prácticas preprofesionales }
\end{array}\right.
$$

COMP (Capacidad de comprensión lectora). Esta variable se mide a través de las veces que el estudiante lee un artículo de su especialidad para dominarlo totalmente. Recibe el valor de 3 si lee una vez, 2 si lee dos veces, 1 si lee tres veces y cero si lee más de tres veces.

EXPLAB (Experiencia laboral). Es una variable dicotómica que recibe el valor de 1 si en el pasado trabajó o efectuó prácticas preprofesionales y 0 en caso contrario.

PERFPRO (Perfil profesional). Es el grado en que el estudiante cree que su perfil y currículum personal calza con la oferta de puestos de trabajo publicados en los medios de comunicación. El valor va de 0 a 3 .

MBTRA (Conocimiento de métodos de búsqueda de trabajo). Variable dicotómica que recibe el valor de 1 si conoce los métodos de búsqueda de trabajo, 3 en caso contrario. 


\section{Juan León Mendoza, Miguel Arias Inga, Richard Roca Garay}

INFORM (Manejo de programas informáticos). Es una variable que indica la cantidad de programas informáticos que maneja el estudiante (aparte del Word, Excel, Power Point). Recibe el valor 0 si no maneja ninguno, 1 si maneja un programa, 2 si maneja dos programas y 3 si maneja más de tres programas.

EMPAT (Empatía personal). Representa la percepción de la proporción de sus compañeros de estudio que le parecen excelentes personas. Recibe el valor de 0 si cree que no hay compañeros excelentes, 1 si es la minoría, 2 si es la mayoría y 3 si cree que todos los compañeros de estudio de la universidad son excelentes personas.

NOT (Promedio ponderado de notas). Es el promedio ponderado de la calificación (nota) alcanzada en el semestre académico anterior. Recibe el valor de 0 si fue menor a 10,1 si el promedio estuvo entre 11-12, 2 si estuvo entre 13-14, 3 si estuvo entre 15-16 y 4 si el promedio obtenido fue más de 16.

RESPON (Grado de responsabilidad personal). Esta variable se aproximó con las veces en que el estudiante llegó tarde a sus clases de la universidad en el último semestre académico. Recibe el valor de 0 si llegó tarde muchas veces, 1 si llegó pocas veces y 2 si nunca llegó tarde.

Dado el ejercicio automático de iteración, los coeficientes asociados a las variables COMP, EXPLAB, PERFPRO, MBTRA, INFORM, EMPAT, NOT y RESPON resultaron ser distinto de 0 , tal como se puede observar en el cuadro $\mathrm{N}^{\circ} .10$. 


\section{Pensamiento Crítico Vol. 19. N I}

\section{CUADRO No 10. PROCESO DE ITERACIÓN Y ESTIMACIÓN ECONOMÉTRICA}

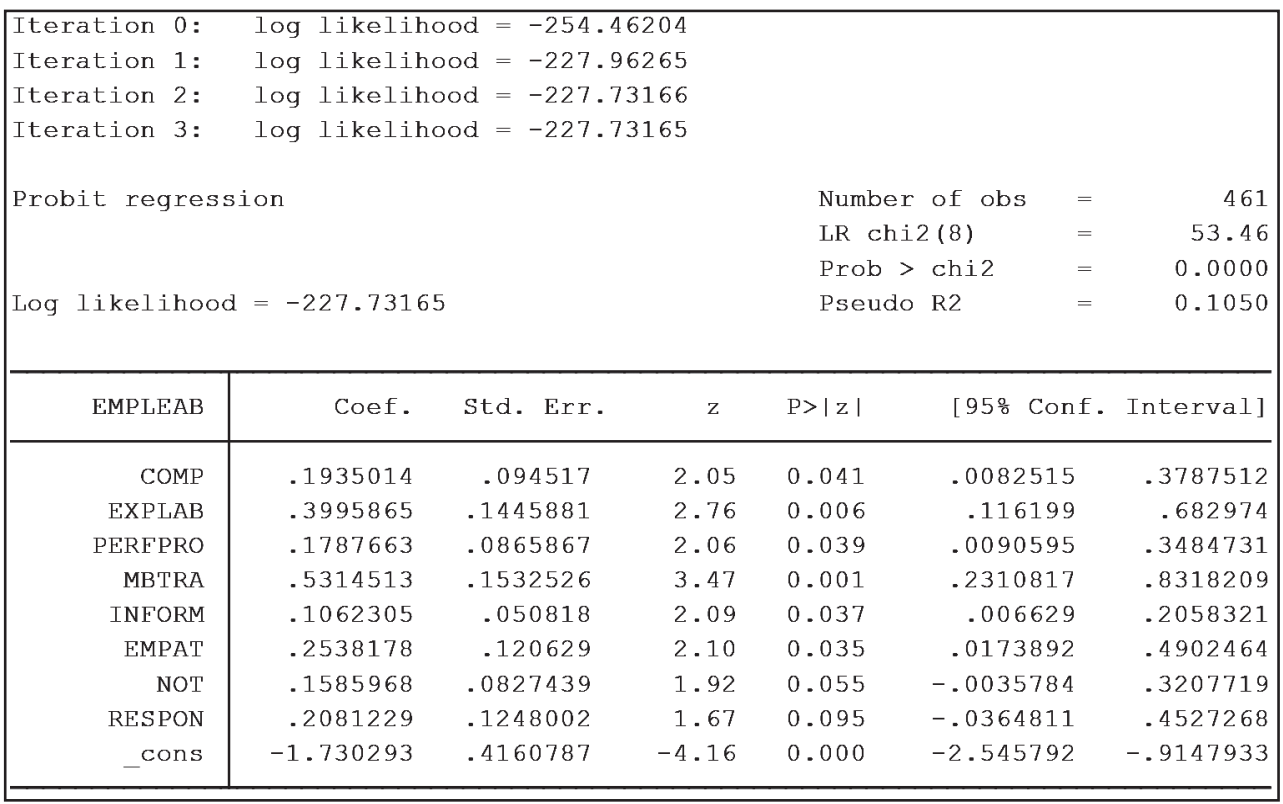

La interpretación de los resultados hallados y expuestos en el cuadro 10 solo se limitan a indicar el sentido del impacto de las variables explicativas sobre la probabilidad de trabajar o realizar práctica preprofesional. Todos los parámetros estimados tienen el signo esperado. Sin embargo, con la finalidad de estimar la magnitud del impacto de las variables independientes especificadas, en especial de las variables dicotómicas, sobre la probabilidad de conseguir empleo, se requiere analizar el efecto marginal correspondiente.

Los resultados de la estimación de los efectos marginales de las variables dicotómicas expuestas en el cuadro 11 indican que la probabilidad potencial de que el estudiante sanmarquino consiga trabajo o una práctica preprofesional es de 0.084 puntos porcentuales mayor si posee alguna experiencia laboral anterior (EXPLAB) que si no la posee. De la misma forma, si el estudiante conoce los métodos de búsqueda de trabajo (MBTRA), su probabilidad de trabajar o realizar prácticas preprofesionales es mayor en 0.0735 puntos porcentuales frente a alguien que no tiene dicho conocimiento. 


\section{Juan León Mendoza, Miguel Arias Inga, Richard Roca Garay}

\section{CUADRO No 11. ESTIMACIÓN DE LOS EFECTOS MARGINALES}

\begin{tabular}{|c|c|c|c|c|c|c|c|}
\hline $\begin{array}{r}\text { Marginal } \\
y= \\
=\end{array}$ & \multicolumn{2}{|c|}{$\begin{array}{l}\text { effects after probit } \\
=\operatorname{Pr}(\text { EMPLEAB) (predict) }\end{array}$} & \multirow[b]{2}{*}{ z } & \multirow[b]{2}{*}{$P>|z|$} & \multirow[b]{2}{*}{$95 \%$} & \multirow[b]{2}{*}{ C.I. } & \multirow[b]{2}{*}{$\mathrm{x}$} \\
\hline variable & $d y / d x$ & Std. Err. & & & & & \\
\hline СОМP & .0317685 & .01715 & 1.85 & 0.064 & -.001854 & .065392 & 0 \\
\hline EXPLAB* & .0840779 & .04882 & 1.72 & 0.085 & -.011609 & .179764 & 0 \\
\hline PEREPRO & .0293494 & .01711 & 1.72 & 0.086 & -.004176 & .062875 & 0 \\
\hline MB'TRA * & .0735058 & .04774 & 1.54 & 0.124 & -.020068 & .16708 & .748373 \\
\hline INFORM & .0174406 & .00997 & 1.75 & 0.080 & -.002105 & .036986 & 0 \\
\hline ЕМРAT & .0416711 & .02129 & 1.96 & 0.050 & -.000056 & .083398 & 0 \\
\hline NOT & .026038 & .01423 & 1.83 & 0.067 & -.001853 & .053929 & 0 \\
\hline RESPON & .0341691 & .02307 & 1.48 & 0.139 & -.01104 & .079378 & 0 \\
\hline
\end{tabular}

Finalmente, estimamos el valor de la elasticidad correspondiente para las demás variables explicativas diferentes a la experiencia laboral y el conocimiento de los métodos de búsqueda de trabajo (variables discretas no dicotómicas). El resultado se muestra en el cuadro 12.

La estimación econométrica efectuada indica que un incremento de $1 \%$ en la comprensión lectora (COMP), el perfil profesional (PERFPRO), el manejo de programas informáticos (INFORM), la empatía personal (EMPAT), el promedio ponderado de notas (NOT) y el nivel de responsabilidad (RESPON) incrementa la probabilidad potencial del estudiante sanmarquino- de que pueda encontrar empleo en $0.12 \%, 0.14 \%, 0.09 \%$, $0.15 \%, 0.14 \%$ y $0.05 \%$, respectivamente.

En resumen, si bien el estudiante promedio de la UNMSM presenta un índice de empleabilidad media, la probabilidad potencial de que este encuentre empleo está determinada fundamentalmente por el hecho de que tenga una mayor capacidad de comprensión lectora, experiencia laboral, una concepción optimista de poseer un buen perfil profesional, un mayor conocimiento de los métodos de búsqueda de empleo, dominio de un mayor número de programas informáticos, una mayor empatía con sus compañeros de estudio, mayores niveles de calificación o promedio de notas y altos niveles de responsabilidad personal. 


\section{Pensamiento Crítico Vol. 19. No I}

\section{CUADRO 12. ESTIMACIÓN DE LAS ELASTICIDADES}

\begin{tabular}{|c|c|c|c|c|c|c|c|}
\hline \multicolumn{8}{|c|}{$\begin{array}{l}\text { Elasticities after probit } \\
\qquad \begin{array}{l}y=\operatorname{Pr} \text { (EMPLEAB) (predict) } \\
=.78251615\end{array}\end{array}$} \\
\hline variable & ey/ex & Std. Err. & $z$ & $P>|z|$ & $95 \%$ & C.I. & $\mathrm{X}$ \\
\hline COMP & .1205417 & .05895 & 2.04 & 0.041 & .005006 & .236077 & 1.65727 \\
\hline EXPIAB & .1042607 & .03796 & 2.75 & 0.006 & .029861 & .17866 & .694143 \\
\hline PERFPRO & .1405149 & .0683 & 2.06 & 0.040 & .006654 & .274376 & 2.09111 \\
\hline MBTRA & .1495004 & .04354 & 3.43 & 0.001 & .064162 & .234839 & .748373 \\
\hline INFORM & .0901695 & .0431 & 2.09 & 0.036 & .005702 & .174638 & 2.25813 \\
\hline EMPAT & .1533557 & .07281 & 2.11 & 0.035 & .010657 & .296054 & 1.60738 \\
\hline NOT & .1395326 & .07267 & 1.92 & 0.055 & -.0029 & .281966 & 2.34056 \\
\hline RESPON & .0459885 & .02761 & 1.67 & 0.096 & -.008117 & .100093 & .587852 \\
\hline
\end{tabular}

\section{Conclusiones}

a) El índice promedio de empleabilidad laboral de los estudiantes de la UNMSM es de $55.51 \%$, que indica que estos presentan, potencialmente, una empleabilidad media en el mercado laboral.

b) Según la regresión econométrica efectuada, la probabilidad potencial de que el estudiante de la UNMSM consiga empleo es mayor en la medida en que tenga una buena capacidad de aprendizaje, posea experiencia laboral, conozca métodos de búsqueda de trabajo, su perfil calce con las necesidades del mercado, domine programas informáticos, alcance altas calificaciones de notas, sea responsable y mantenga alta empatía con sus compañeros de la universidad.

\section{Referencias bibliográficas}

ALLEN, Jim, Ger RAMAEKERS y Rolf VAN DER VELDEN (2003). La medición de las competencias de los titulados superiores. Centro de Investigación para la Educación y el Mercado Laboral Universidad de Maastricht, Países Bajos. Publicado en Métodos de análisis de la inserción laboral de los universitarios, Javier Vidal García Coordinador; Salamanca España, 2003. Disponible en: http://200.6.99.248/ bru487cl/files/libros/ Tendencias/pdf/Insercion_laboral.pdf 


\section{Juan León Mendoza, Miguel Arias Inga, Richard Roca Garay}

COLLADO, Andrea (2006) Construcción de un indicador para medir competencias básicas para la vida. Población y Salud en Mesoamérica, Vol. 3, No. 2, Costa Rica. Disponible en: http://ccp.ucr.ac.cr/revista/

CORPOEDUCACIÓN (2003). Competencias laborales: base para mejorar la empleabilidad de las personas, Bogotá. Disponible en: http://www.colombiaaprende. edu.co/html/mediateca/1607/articles-101815_archivo.pdf

FORRIER, Anneleen y Luc SELS (2003). The concept employability: a complex mosaic. International Journal of Human Resources Development and Management. Vol. 3, Disponible en: http:/www.ouderenenarbeid.be/documenten/artikel\%20ijhrdm.pdf

FUNDIPE (2010). Empleabilidad. Informe, Madrid. Disponible en: http://www.fundipe. es/archives/INFORMEE_Seguro.pdf

FUNDACIÓN GAZTELÁN (2007). Diagnóstico de empleabilidad. Disponible en:http://www.sieres.org/integracion/orientacion-e-intervencion-sociolaboral/ empleabilidad_51_1_ap_basica.html

HARVEY, Lee (1999) Employability: developing the relationship between higher education and employment. Scarman House, Warwick University. Citada por Rodríguez (2009).

PUENTE, Esteban y Tomás RAU (2010). Elaboración de un índice de empleabilidad y vulnerabilidad laboral. Informe final. PNUD, Santiago de Chile. Disponible en: http://www.pnud.cl/proyectos/Documentos\%20de\%20proyectos/2011/Informe\%20 Final_010210.pdf

RENTERÍA, Erico y Verónica ANDRADE (2007) Representaciones y acciones de los alumnos universitarios frente a la empleabilidad. Psicología del Caribe, No. 20. Universidad del Norte. Disponible en: http://ciruelo.uninorte.edu.co/pdf/psicologia caribe/20/6_Representaciones\%20y\%20acciones.pdf 


\section{Pensamiento Crítico Vol. 19. No I}

RODRÍGUEZ, Javier (2009). Índice de empleabilidad de los jóvenes. Serie Avances de Investigación No. 32. CISEPA, Pontificia Universidad Católica del Perú, Lima. Disponible en: http:/www.fundacioncarolina.es/es-ES/publicaciones/ avancesinvestigacion/Documents/AI32\%20Empleabilidad\%20j\%C3\%B3venes.pdf

RODRÍGUEZ, Sebastián, Anna PRADES, Lorena BERNÁLDEZ y Sergio SÁNCHEZ (2010). Sobre la empleabilidad de los graduados universitarios en Catalunya: del diagnóstico a la acción. Revista de Educación No. 351, Barcelona, España. Disponible en: http://www.revistaeducacion.mec.es/re351_05.html.

UNIÓN EUROPEA (2010) Plan de mejora de la empleabilidad del municipio de Velez Málaga 2011-2013. Disponible en: http://ebookbrowsee.net/reducido-fase-1diagnostico-del-plan-de-movilidad-sostenible-de-velez-malaga-pdf-d496430238 